\title{
Research Paper: The Effects of Negative Heel Rocker Shoes on the Moment and the Contact Forces Applied on Lower Limb Joints of Diabetic Patients During Walking
}

\author{
Keyvan Sharifmoradi ${ }^{1 *}$, Mohammad Taghi Karimi², Zeynab Rezaeeyan² \\ 1. Department of Physical Education, Faculty of Humanities, University of Kashan, Kashan, Iran. \\ 2. Department of Orthotics and Prosthetics, Musculoskeletal Research Center, School of Rehabilitation Sciences, Isfahan University of Medical Sciences, Isfahan, Iran.
}

Citation: Sharifmoradi K, Karimi MT, Rezaeeyan Z. The Effects of Negative Heel Rocker Shoes on the Moment and the Contact Forces Applied on Lower Limb Joints of Diabetic Patients During Walking. Physical Treatments. 2016; 6(3):129-136. https://doi.org/10.18869/nrip.ptj.6.3.129

dol: : https://doi.org/10.18869/nrip.ptj.6.3.129

Article info:

Received: 06 Jul. 2016

Accepted: 02 Sep. 2016

Keywords:

Type 2 diabetes mellitus, Heel rocker shoe, Walking, Joint contact force, Moment

\begin{abstract}
A B S T R A C T
Purpose: The negative heel rocker shoes help reduce the plantar pressure in patients with diabetes, but their effects on the other lower limb joints are unknown. Accordingly, the current study aimed at evaluating the effect of negative heel rocker shoes on the moment and the contact forces applied on lower limb joints of diabetic patients while walking.

Methods: A total of 10 patients with diabetes mellitus and 10 healthy individuals were enrolled to this quasi-experimental study. Musculoskeletal system modeling was initiated using OpenSIM and Visual 3D. After noise elimination and data processing, the independent $t$ test was done. The statistical analyses were done by SPSS 19 and significance level was considered at less than 0.05 .

Results: Vertical contact force of hip joint in the experimental group (1.17 Newton/body weight) was significantly higher than that of the control group $(\mathrm{P}=0.04)$. In the experimental group, the anterior-posterior contact forces of hip joint $(\mathrm{P}=0.01)$, knee joint $(\mathrm{P}=0.01)$, and ankle joint $(\mathrm{P}=0.04)$ were $1.3,1.5$, and $0.47 \mathrm{~N} / \mathrm{BW}$, respectively, which were significantly higher than those of the control group. In the experimental group, the internal-external contact forces of hip joint $(\mathrm{P}=0.01)$ and knee joint $(\mathrm{P}=0.01)$ were 1.31 and $0.15 \mathrm{~N} / \mathrm{BW}$, respectively; which were significantly lower than those of the control group.
\end{abstract}

Conclusion: Using negative heel rocker shoes is associated with decrease in the vertical contact force of ankle joint. Heel rocker shoes increase vertical and anterior-posterior forces of proximal lower limb joints (hip and knee). The increased contact force in proximal lower limb joints, especially hip joint, may increase the risk of joint injury and disruption. Thus, more attention should be paid to prescribe negative heel rocker shoes for the patients with diabetes.

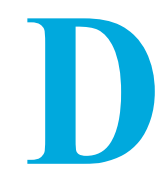

\section{Introduction}

iabetic foot ulcer can cause irrecoverable consequences such as amputation and death, which impose heavy costs to the patient and the community [1]. Prescription of suitable shoes and insoles reduces the plantar pressure and prevents the diabetic foot ulceration and or re-ulceration [2], desirably

* Corresponding Author:

Keyvan Sharifmoradi, $P h D$

Address: Department of Physical Education, Faculty of Humanities, University of Kashan, Kashan, Iran.

Phone: +98 (913) 8160103

E-mail: ksharifmoradi@gmail.com 
increases the quality of life, and decreases health care services [3]. Several studies indicate the significant and positive effects of diabetic shoes on decreasing the plantar pressure [4-6] and show that metatarsal ulcers reduce by using suitable shoes $[7,8]$. Thus, these shoes reduce pressures imposed on feet and are a remarkable mediator in the prevention of re-ulceration [9].

Ulcers usually occur in high pressure metatarsal regions such as under the fingers, under the first, fifth, and second to fourth metatarsal heads $[10,11]$. The rocker shoes could reduce the plantar pressure and subsequently treat diabetic foot ulcers $[11,12]$. One of the most common types of these shoes is the heel rocker ones. The heel rocker shoes are designed to bear the weight, transfer it to backward, reduce the problems of middle plantar region, and also decrease shock and impulse while lifting the foot [13].

Forghani et al. reported that rocker shoes could reduce the ankle moment after heel contact and decrease plantar pressure under the heel and transfer it to the middle plantar [14]. Long et al. evaluated the kinetic and kinematic effects of rocker shoes on hip joints, and reported that reduction in internal rotation moment at heel contact would increase the power absorption in the middle standing phase and increase the power generation in the initial swing phase. They also showed that the power generation increased from the mid-stance phase to the beginning of the swing phase in the knee joint, and the power absorption decreased in the beginning of the stance phase in ankle joint [15].

In another study, Madden et al. reported that knee adductor moment would reduce during walking with wearing heel rocker shoes, compared with the regular shoes. Hence, wearing heel rocker shoes can reduce internal knee forces [16]. Researchers evaluated the effect of heel rocker shoes on the kinematics and kinetics of lower limbs joints and reported that heel rocker shoes mostly affect proximal joints (knee and hip) rather than distal joints (ankle); they also showed that the changes that occur in the transverse plane are more than those of sagittal and frontal planes [17].

As indicated before, most recently conducted studies have reported the reduction of plantar pressure in the patients with diabetes through the intervention of rocker shoes and recommend the patients with diabetes to use such shoes. The previously conducted studies evaluated the mechanism of plantar pressure changes while using rocker shoes, but neglected the effect of the shoes on the joints of upper limbs. The studies reported that the reduc- tion of plantar pressure through the intervention of rocker shoes in a region of foot was associated with plantar pressure increase in another region of the foot [14]. This plantar pressure change from one region to another can change the joint contact force in the lower limbs through changing the moment [16].

In case of applying improper forces over the joint, the joint may be at the risk of disruption. Thus prescription of rocker shoes for the patients with diabetes and special status may cause diabetic ulceration in unexpected regions neglected by the specialists and cause irreparable consequences. More information and knowledge about the mechanism of force changes can improve and promote the attitudes toward high risk factors in the joints of lower limbs. Hence, recognition of the joints which remain at high risk of overload, following the use of rocker shoes, is of great importance. To the best of author's knowledge, no study has evaluated the forces applied to the joints of patients with diabetes following the use of heel rocker shoes. In addition, contrary to the findings of other studies, which calculated the joint reaction force through inverse dynamics, the current study calculated the joint contact forces through OpenSIM modelling, including a sum of all joint reaction forces and muscular forces. The human gait simulation musculoskeletal model can anticipate the muscular activity and contact joint force patterns, and benefits from high accuracy in different gait speeds $[18,19]$. Therefore, the current study aimed at evaluating the effect of heel rocker shoes on the contact forces applied on the joints of lower limbs in patients with diabetes while walking.

\section{Materials and Methods}

The current quasi-experimental study was conducted in the Faculty of Rehabilitation, Isfahan University of Medical Sciences, Isfahan, Iran, in 2014. A total of 10 patients with diabetes and neuropathy, with the average (SD) height, weight, and foot length of $153.10(3.63) \mathrm{cm}$, $84.59(14.53) \mathrm{kg}$, and $73.50(2.50) \mathrm{cm}$, respectively, and also 10 healthy participants with the average (SD) height, weight, and foot length of $153.10(6.17) \mathrm{cm}, 63.24(20.39)$ $\mathrm{kg}$, and 78.80(3.22) $\mathrm{cm}$, respectively, were enrolled to the study. The subjects were selected out of the patients referred to the specialized clinic and health care centers of Isfahan, Iran, by convenience sampling method and considering the inclusion criteria. The inclusion criteria were as follows: aged between 30 and 60 years $[13,15,17,20]$, diagnosed with diabetic neuropathy $[13,15,17,20]$, ability to bear plantar overweight and pressure [13, 21], and ability to walk independently without aids [13, 20, 21]. The exclusion criteria were as follows: lack of diabetic 


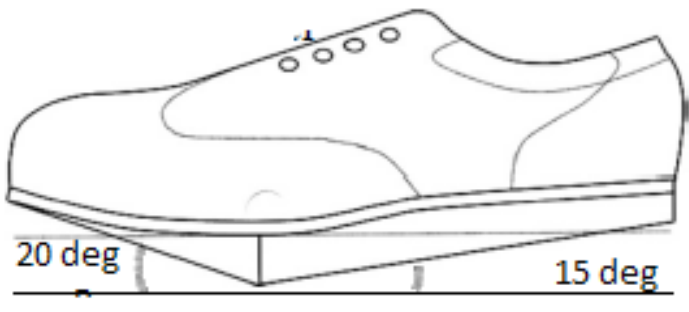

PHYSICAL TREA T MENTS

Figure 1. The heel rocker embedded in the shoe

neuropathy, development of any plantar ulceration or deformation [15, 17, 20, 21], and underwent any surgical procedures within 6 months prior to the study [13, 20, 21].

\section{Specification of shoes and rockers}

The shoes were made of medically standard leather by an orthopedist in a shoe workshop, without rocker and sole. The heel rocker was designed and made by the orthopedist. High density foam was used for the heel rocker from heel to head with an angle of $20^{\circ}$ between the head and anterior part of the rocker, and an angle of $15^{\circ}$ between the interior part of rocker and ground surface (Figure 1) [22].

After explaining the study purposes to the participants, they signed the written consents. Before data collection, the subjects were familiar with the experiment procedure; in a way that each subject was asked to repeat the gait test for 5 times to get familiar with the procedure. Then, the experiment was conducted.

\section{Measurement instrument}

To measure the kinematic gait variables, Qualisys motion analysis system (Qualisys Co., USA), equipped with 7 cameras, was used. The cameras were embedded on both sides and the center of the force plate. A $10-\mathrm{m}$ pavement was established along the laboratory and a Kistler force plate $(500 \times 600 \mathrm{~mm}$, AA 9260 model $)$ (Kistler Co., Swiss) was placed at its center. The accuracy of dynamometer was really high with the error rate below $1 \%$ [23].

Twenty infrared-reflecting markers with $14 \mathrm{~mm}$ diameter were fixed to the anterior-superior and posterior-superior of iliac spine, interior and exterior epicondyles on right and left sides of feet, heel, the first and fifth metatarsal heads, and acromioclavicular joint on right and left sides at sitting position. The markers fixing areas coincided with the protocol approved by Strathclyde University. Data collection frequency was $120 \mathrm{~Hz}$; data were filtered using a $10 \mathrm{~Hz}$ low pass filter. The kinematic data were recorded by Qualisys Track Manager version 2.7 (Qualisys Co., USA). Also, the musculoskeletal system of the subject was modelled by Visual3D version 4 (C-motion Co., USA). The data processed in Visual3D were transferred to OpenSIM version 3 (Stanford University production, USA) to evaluate the contact force of the joints of lower limbs. OpenSIM is a simulator and musculoskeletal system analyzer, which can analyze and estimate the joint contact force and the muscle forces. Table 1 shows the results of data analysis by OpenSIM. By stimulation of musculoskeletal system, the diagnosis and treatment of abnormal and pathologic motions were provided [1].

After calibrating the cameras and force plate, anthropometric data of the study subjects, including weight, height, and right and left feet length were recorded. Then, the static test was conducted and afterward, the subjects walked on the pavement and the images of the markers along with the force plate data were recorded. The standing and swing-gait data of the phases were recorded by Qualisys.

\section{Gait test}

Test procedure and required trainings were given to the subjects in an orientation session. After calibrating the cameras, fixing the markers, and performing the static test at the center of calibrated zone, the subjects were asked to walk with the designed rocker. The healthy subjects were also asked to perform the test with medio-lat-

Table 1. Data extraction stages in openSIM 
eral. The subject's gait test was repeated 5 times and the mean repetition was set to 5 for all variables to analyze the data [24]. To prevent subjects' fatigue, 30 seconds relaxation was considered between the tests repetition [25].

\section{Data analysis}

All data recorded for each subject were divided by his body weight to be normalized. The normal distribution of parameters was evaluated by the Shapiro-Wilk test. Data analysis was conducted using the independent $t$ test by SPSS 22 (SPSS Inc., Chicago, IL, USA); $\mathrm{P}<0.5$ was considered as level of significance.

\section{Results}

Table 2 presents the mean (SD) values of the moment applied on different joints of the lower limbs. Accordingly, a significant difference was observed between the experimental and control groups regarding the extension moment of knee joint $(\mathrm{P}=0.03)$ and hip joint $(\mathrm{P}=0.00)$. The extension moment of hip and knee joints while walking with heel rocker shoes were 1.07 and $0.40 \mathrm{Nm} /$ $\mathrm{BW}$, respectively, which were significantly higher than those of barefoot walking in the control group as 0.47 and $0.09 \mathrm{Nm} / \mathrm{BW}$, respectively. Also, the plantarflexion moment of the experimental group was $1.24 \mathrm{Nm} / \mathrm{BW}$, which was more than that of the control group as 0.17 $\mathrm{Nm} / \mathrm{BW}(\mathrm{P}=0.22)$, but the difference between the groups was insignificant. The moments for the rest of the joints of lower limbs showed no significant difference between the experimental and control groups.

Table 3 presents the mean (SD) values of contact forces of lower limb joints. There were significant differences between the experimental and control groups regarding the anterior-posterior $(\mathrm{P}=0.00)$, vertical $(\mathrm{P}=0.04)$, and medio-lateral $(\mathrm{P}=0.00)$ contact force of hip joint. The anterior-posterior joint contact force in the experimental group was $1.93 \mathrm{~N} / \mathrm{BW}$, which was approximately 3 times greater than that of the control group. The vertical hip joint contact force in the experimental group was $5.76 \mathrm{~N} / \mathrm{BW}$, which was $1.17 \mathrm{~N} / \mathrm{BW}$ greater than that of the control group. The medio-lateral hip joint contact force in the experimental group was $0.16 \mathrm{~N} / \mathrm{BW}$, which was $1.04 \mathrm{~N} / \mathrm{BW}$ greater than that of the control group.

There were significant differences between the experimental and control groups regarding the anterior-posterior $(\mathrm{P}=0.00)$ and medio-lateral $(\mathrm{P}=0.00)$ knee joint contact forces. The anterior-posterior knee joint contact force in the rocker shoes group was 1.5 N/BW greater than that of the control group; while, the medio-lateral knee joint contact force in the rocker shoes group was $0.15 \mathrm{~N} / \mathrm{BW}$ lower than that of the control group. There was no significant difference between the rocker shoes and control groups regarding the vertical knee joint contact force $(\mathrm{P}=0.59)$.

There was a significant difference between the experimental and control groups regarding the anterior-posterior $(\mathrm{P}=0.04)$ and vertical $(\mathrm{P}=0.03)$ ankle joint contact forces. The anterior-posterior contact force of the experimental group was $1.56 \mathrm{~N} / \mathrm{BW}$, which was $0.47 \mathrm{~N} / \mathrm{BW}$ greater than that of the control group; while, the vertical ankle joint contact force in the experimental group was $1.58 \mathrm{~N} / \mathrm{BW}$ greater than that of the control group. There was no significant difference between two groups regarding the medio-lateral contact force $(\mathrm{P}=0.30)$.

\section{Discussion}

The current study aimed at evaluating the effect of heel rocker shoe on the moment and contact forces applied on the lower limbs joints in patients with diabetes during walking. The results indicated that the ankle and knee moments in the sagittal plane of the experimental group

Table 2. Mean (SD) values of the moment applied on different joints of the lower limbs.

\begin{tabular}{|c|c|c|c|c|}
\hline & Experimental Group & Control Group & $\mathbf{t}$ & $\mathbf{P}$ \\
\hline Dorsi flexion & $0.08 \pm 0.1$ & $0.09 \pm 0.11$ & 0.38 & 0.70 \\
\hline Plantar flexion & $1.24 \pm 0.20$ & $1.70 \pm 0.75$ & 1.24 & 0.22 \\
\hline Knee flexion & $0.37 \pm 0.28$ & $0.26 \pm 0.16$ & 1.80 & 0.08 \\
\hline Knee extension & $0.40 \pm 0.13$ & $0.31 \pm 0.15$ & 2.28 & $0.03 *$ \\
\hline Hip flexion & $0.49 \pm 0.24$ & $0.6 \pm 0.42$ & 1.21 & 0.23 \\
\hline Hip extension & $1.07 \pm 0.24$ & $0.6 \pm 0.22 *$ & 7.56 & $0.01^{*}$ \\
\hline
\end{tabular}

* The significant difference 
Table 3. The mean (SD) of contact force of lower limbs joints.

\begin{tabular}{|c|c|c|c|c|c|}
\hline & Joint Contact Force & Experimental Group & Control Group & $\mathbf{t}$ & $\mathbf{P}$ \\
\hline \multirow{3}{*}{ Hip } & Anterior-posterior & $1.93 \pm 0.65$ & $0.63 \pm 0.21$ & 6.07 & $0.01^{*}$ \\
\hline & Vertical & $5.76 \pm 1.52$ & $4.59 \pm 0.77$ & 2.16 & $0.04^{*}$ \\
\hline & medio-lateral & $1.52 \pm 0.05$ & $1.21 \pm 0.28$ & 11.80 & $0.01^{*}$ \\
\hline \multirow{3}{*}{ Knee } & Anterior-posterior & $2.31 \pm 1.14$ & $0.8 \pm 0.49$ & 3.84 & $0.01 *$ \\
\hline & Vertical & $4.32 \pm 0.96$ & $4.11 \pm 0.71$ & 0.54 & 0.59 \\
\hline & medio-lateral & $0.10 \pm 0.07$ & $0.25 \pm 0.07$ & 4.82 & $0.01 *$ \\
\hline \multirow{3}{*}{ Ankle } & Anterior-posterior & $1.56 \pm 0.39$ & $1.10 \pm 0.52$ & 2.23 & $0.04^{*}$ \\
\hline & Vertical & $5.78 \pm 2.35$ & $7.36 \pm 1.61$ & 2.38 & $0.03^{*}$ \\
\hline & medio-lateral & $0.54 \pm 0.31$ & $0.42 \pm 0.18$ & 0.52 & 0.30 \\
\hline
\end{tabular}

* The significant difference

PHYSICAL TREA † MENTS

was significantly lower than those of the control group. The vertical contact force of hip joint in the heel rocker shoe group was higher than that of the control group; while, the ankle joint contact force of the heel rocker shoe group was lower than that of the control group.

There was a significant difference between the experimental and control groups with regard to the extension moment of knee and hip joints. The extension moment of knee and hip joints while walking with the heel rocker shoe was $0.14 \mathrm{Nm} / \mathrm{BW}$, which was $0.47 \mathrm{Nm} / \mathrm{BW}$ greater than the moment of barefoot walking in the control group. The plantarflexion moment in the experimental group was $1.22 \mathrm{Nm} / \mathrm{BW}$, which had no significant difference with that of the control group $(\mathrm{P}=0.68)$. Particularly, while walking at the end of stance phase, in the push off stage, the external dorsiflexion moment (ground reaction force $\mathrm{x}$ external moment arm) creates a balance force with the plantar flexor moment (muscle force $\mathrm{x}$ internal moment arm). Changing shoes affects the joint moment. From the biomechanical viewpoint, the rocker shoes with the apex proximal to metatarsophalangeal joint can reduce the external dorsiflexion moment arm, and this reduction result in the reduction of plantarflexion moment around the ankle, which finally reduces the force applied by triceps [25].

Also, previous studies report that the heel rocker shoes cause delay in lifting the heel, reduce the height of heel during walking, and also delay in the progression of pressure. These factors reduce the plantarflexion moment at the end of stance phase [26-28]. According to the results, no significant difference was observed between the heel rocker and control groups regarding the plantar flexor moment. It may result from placing heel rocker shoe with apex under the metatarsophalangeal joint which caused no changes in the external dorsiflexion moment arm and accordingly, no tangible change was occurred in the plantar flexion moment around the ankle joint. Results of the current study was consistent with those of the previous studies $[28,29]$, and inconsistent with the studies which reported that the rocker shoes could reduce plantarflexion moment in the ankle [15, 25, 30, 31].

Analysis of contact forces of lower limbs joints through OpenSIM modeling indicated that the anterior-posterior hip joint contact force in the experimental group was approximately 3 times more than that of the control group. The vertical contact force of hip joint in the experimental group was $1.17 \mathrm{~N} / \mathrm{BW}$ more than that of the control group. Also, analysis of the muscular moment showed that the hip extensor muscles moment during walking with the heel rocker shoes was significantly higher than that of the barefoot walking, indicating the increase in anterior-posterior and vertical joint contact forces in the heel rocker shoes group, compared with those of the control group.

The vertical contact force of hip joint in the experimental group was significantly more than that of the control group, and the vertical contact force of ankle joint in the experimental group was significantly lower than that of the control group.

The anterior-posterior contact forces of ankle, knee, and hip joints in the experimental group were significantly more than those of the control group and the highest anterior-posterior contact force was observed in the hip joint, which was 3 times higher than that of the 
control group. The medio-lateral contact forces of knee and hip joints were significantly reduced by wearing heel rocker shoes, compared with the control group. Hence, the heel rocker shoes can even change the force distribution pattern in joints, increase the anterior-posterior force in the ankle, knee, and hip joints, and significantly reduce medio-lateral forces of knee and hip joints.

Reduction in the plantarflexion moment of ankle can be attributed to lower involvement of muscles crossing the ankle, following the use of rocker shoes. The direct effect of such mechanism is the lower activity of agonist muscles and higher activity of antagonist muscles, which finally reduce the overload on the joints [32, 33]. The heel rocker shoes stimulate the muscles close to the rotation axis of ankle. When the big muscles, which are far from the joint, provide the stability, the load-bearing capacity of the joint increases, and when the stability is provided by the smaller muscles, which are closer to the joint axis, the joints show faster reaction and their loadbearing capacity reduces [29].

The current study results indicated that the vertical loading over the ankle joint in the heel rocker group was significantly lower than that of the control group. It may be due to higher stress in the muscles that are close to the joint axis. Our study results also showed that the reduction of loading over ankle joint occurs in the vertical contact force of the joint and the reduction of loading over knee and hip joints occur in the medio-lateral force of the joints, while the anterior-posterior joint contact force increased in ankle, knee and hip joint; so that the anteriorposterior force of hip joint in the experimental group was 3 times higher than that of the control group. It seems that by wearing heel rocker shoes, the load-bearing capacity of the joint rises along with moving from ankle joint to other proximal joints, which may increase the risk of joint injury and disruption. Since the heel rocker shoes are prescribed for the diabetes to prevent foot ulceration, using such shoes can reduce the plantar pressure and transfer the pressure to the hip joint. Therefore the long-term use of the shoes may cause irreparable consequences for the patient. In this regard, it is recommended that heel rocker shoes be prescribed for the patients with diabetes with more caution, especially for the ones with hip and knee joints abnormalities.

Using heel rocker shoes is associated with reduction in the vertical contact force in the ankle joint. The heel ankle shoes increase the vertical and anterior-posterior contact forces in the proximal joints of lower limbs. Accordingly, the contact force increases in the proximal joints of lower limbs, especially the hip joint, and can in- crease the risk of joint injury and disruption. Therefore, it is necessary to pay more attention in the prescription of heel rocker shoes for the patients with diabetes.

\section{Acknowledgments}

The authors gratefully acknowledge financial support from the Musculoskeletal Research Center of Isfahan University of Medical Sciences.

\section{Conflict of Interest}

The authors report no conflicts of interest.

\section{References}

[1] Ramsey SD, Newton K, Blough D, McCulloch DK, Sandhu $\mathrm{N}$, Reiber GE, et al. Incidence, outcomes, and cost of foot ulcers in patients with diabetes. Diabetes Care. 1999; 22(3):3827. doi: $10.2337 /$ diacare.22.3.382

[2] Bakker K, Schaper NC. The development of global consensus guidelines on the management and prevention of the diabetic foot 2011. Diabetes/Metabolism Research and Reviews. 2012; 28:116-8. doi: 10.1002/dmrr.2254

[3] Ragnarson Tennvall G, Apelqvist J. Health-economic consequences of diabetic foot lesions. Clinical Infectious Diseases. 2004; 39(2):132-9. doi: 10.1086/383275

[4] Adler AI, Boyko EJ, Ahroni JH, Smith DG. Lower-extremity amputation in diabetes: The independent effects of peripheral vascular disease, sensory neuropathy, and foot ulcers. Diabetes Care. 1999; 22(7):1029-35. doi: 10.2337/diacare.22.7.1029

[5] Pecoraro RE, Reiber GE, Burgess EM. Pathways to diabetic limb amputation: Basis for prevention. Diabetes Care. 1990; 13(5):513-21. doi: 10.2337/diacare.13.5.513

[6] Viswanathan V, Madhavan S, Gnanasundaram S, Gopalakrishna G, Das BN, Rajasekar S, et al. Effectiveness of different types of footwear insoles for the diabetic neuropathic Foot: A follow-up study. Diabetes Care. 2004; 27(2):474-7. doi: 10.2337/diacare.27.2.474

[7] Singh N. Preventing foot ulcers in patients with diabetes. Journal of American Medical Association (JAMA). 2005; 293(2):217. doi: 10.1001/jama.293.2.217

[8] Dorresteijn JAN, Valk GD. Patient education for preventing diabetic foot ulceration. Diabetes/Metabolism Research and Reviews. 2012; 28:101-6. doi: 10.1002/dmrr.2237

[9] Owings TM, Apelqvist J, Stenström A, Becker M, Bus SA Kalpen A, et al. Plantar pressures in diabetic patients with foot ulcers which have remained healed. Diabetic Medicine. 2009; 26(11):1141-6. doi: 10.1111/j.1464-5491.2009.02835.x

[10] Cavanagh PR, Simoneau GG, Ulbrecht JS. Ulceration, unsteadiness, and uncertainty: The biomechanical conse- 
quences of diabetes mellitus. Journal of Biomechanics. 1993; 26:23-40. doi: 10.1016/0021-9290(93)90077-r

[11] Janisse DJ, Coleman W. Pedorthic care of the diabetic foot: Correlation with risk category. Levin and O'Neal's The Diabetic Foot. 2008; 529-46. doi: 10.1016/b978-0-323-041454.50035-1

[12] White J. Therapeutic footwear for patients with diabetes. Journal of the American Podiatric Medical Association. 1994; 84(9):470-9. doi: 10.7547/87507315-84-9-470

[13] Brown D, Wertsch JJ, Harris GF, Klein J, Janisse D. Effect of rocker soles on plantar pressures 11 No commercial party having a direct financial interest in the results of the research supporting this article has or will confer a benefit upon the author(s) or upon any organization with which the author(s) is/are associated. Archives of Physical Medicine and Rehabilitation. 2004; 85(1):81-6. doi: 10.1016/s00039993(03)00374-5

[14] Forghany S, Nester C, Richards B, Hatton A. Effect of rollover footwear on metabolic cost of ambulation, lower limb kinematics, kinetics, and EMG related muscle activity during walking. Journal of Foot and Ankle Research. 2012; 5(1):O4. doi: 10.1186/1757-1146-5-s1-o4

[15] Long JT, Klein JP, Sirota NM, Wertsch JJ, Janisse D, Harris GF. Biomechanics of the double rocker sole shoe: Gait kinematics and kinetics. Journal of Biomechanics. 2007; 40(13):2882-90. doi: 10.1016/j.jbiomech.2007.03.009

[16] Madden EG, Kean CO, Wrigley TV, Bennell KL, Hinman RS. Effects of unstable rocker-soled shoes on knee load parameters in people with knee osteoarthritis. Osteoarthritis and Cartilage. 2012; 20:97-8. doi: 10.1016/j.joca.2012.02.103

[17] Myers KA, Long JT, Klein JP, Wertsch JJ, Janisse D, Harris GF. Biomechanical implications of the negative heel rocker sole shoe: Gait kinematics and kinetics. Gait \& Posture. 2006; 24(3):323-30. doi: 10.1016/j.gaitpost.2005.10.006

[18] Modenese L, Phillips ATM. Prediction of hip contact forces and muscle activations during walking at different speeds. Multibody System Dynamics. 2011; 28(1-2):157-68. doi: 10.1007/s11044-011-9274-7

[19] Wallace JF. Gait mechanics, joint contact forces, and muscle forces in older adults with radiographic knee osteoarthritis and knee pain compared to a similar population with no radiographic knee osteoarthritis [PhD dissertation]. WinstonSalem: Wake Forest University; 2014.

[20] Owings TM, Woerner JL, Frampton JD, Cavanagh PR, Botek G. Custom therapeutic insoles based on both foot shape and plantar pressure measurement provide enhanced pressure relief. Diabetes Care. 2008; 31(5):839-44. doi: 10.2337/ dc07-2288

[21] Reiber GE, Smith DG, Wallace C, Sullivan K, Hayes S, Vath C, et al. Effect of therapeutic footwear on foot reulceration in patients with diabetes. Journal of American Medical Association (JAMA). 2002; 287(19):2552. doi: 10.1001/ jama.287.19.2552

[22] Van Schie C, Ulbrecht JS, Becker MB, Cavanagh PR. Design criteria for rigid rocker shoes. Foot \& Ankle International. 2000; 21(10):833-44. doi: 10.1177/107110070002101007
[23] Hall MG, Fleming HE, Dolan MJ, Millbank SFD, Paul JP. Static in situ calibration of force plates. Journal of Biomechanics. 1996; 29(5):659-65. doi: 10.1016/0021-9290(95)00109-3

[24] Keselman HJ, Othman AR, Wilcox RR, Fradette K. The new and improved two-sample $t$ test. Psychological Science. 2004; 15(1):47-51. doi: 10.1111/j.0963-7214.2004.01501008.x

[25] Sobhani S, Hijmans J, van den Heuvel E, Zwerver J, Dekker $\mathrm{R}$, Postema K. Biomechanics of slow running and walking with a rocker shoe. Gait \& Posture. 2013; 38(4):998-1004. doi: 10.1016/j.gaitpost.2013.05.008

[26] Turner DE, Woodburn J. Characterising the clinical and biomechanical features of severely deformed feet in rheumatoid arthritis. Gait \& Posture. 2008; 28(4):574-80. doi: 10.1016/j.gaitpost.2008.04.004

[27] Turner DE, Helliwell PS, Siegel KL, Woodburn J. Biomechanics of the foot in rheumatoid arthritis: Identifying abnormal function and the factors associated with localised disease "impact". Clinical Biomechanics. 2008; 23(1):93-100. doi: 10.1016/j.clinbiomech.2007.08.009

[28] Schmiegel A, Rosenbaum D, Schorat A, Hilker A, Gaubitz M. Assessment of foot impairment in rheumatoid arthritis patients by dynamic pedobarography. Gait \& Posture. 2008; 27(1):110-4. doi: 10.1016/j.gaitpost.2007.02.008

[29] Nigg B, Hintzen S, Ferber R. Effect of an unstable shoe construction on lower extremity gait characteristics. Clinical Biomechanics. 2006; 21(1):82-8. doi: 10.1016/j.clinbiomech.2005.08.013

[30] Landry SC, Nigg BM, Tecante KE. Walking in an unstable Masai Barefoot Technology (MBT) shoe introduces kinematic and kinetic changes at the hip, knee and ankle before and after a 6-week accommodation period: A comprehensive analysis using Principal Component Analysis (PCA). Footwear Science. 2012; 4(2):101-14. doi: $10.1080 / 19424280.2012 .684448$

[31] Taniguchi M, Tateuchi H, Takeoka T, Ichihashi N. Kinematic and kinetic characteristics of Masai Barefoot Technology footwear. Gait \& Posture. 2012; 35(4):567-72. doi: 10.1016/j. gaitpost.2011.11.025

[32] Andriacchi TP, Natarajan RN, Hurwitz DE. Musculoskeletal dynamics, locomotion, and clinical applications. Basic Orthopaedic Biomechanics. 1997; 2:37-68.

[33] Herzog W, Longino D, Clark A. The role of muscles in joint adaptation and degeneration. Langenbeck's Archives of Surgery. 2003; 388(5):305-15. doi: 10.1007/s00423-003-0402-6 
October 2016. Volume 6. Number 3

PHYSICAL TREATMENTS 